УДК 621.313.282.2

\title{
ОБОБЩЕННАЯ МОДЕЛЬ ДВУХКАТУШЕЧНОЙ СИНХРОННОЙ ЭЛЕКТРОМАГНИТНОЙ МАШИНЫ ДЛЯ ТЕХНОЛОГИЧЕСКИХ СИСТЕМ ВИБРОУДАРНОГО ДЕЙСТВИЯ
}

\author{
Нейман Людмила Андреевна', \\ neyman31@gmail.com \\ Нейман Владимир Юрьевич1, \\ nv.nstu@ngs.ru \\ 1 Новосибирский государственный технический университет, \\ Россия, 630073, г. Новосибирск, пр. Карла Маркса, 20.
}

\begin{abstract}
Актуальность исследования обусловлена тем, что силовые электрические импульсные системы, созданные на основе линейных электромагнитных машин ударного и виброударного действия, являются перспективными для применения в технологиях по интенсификации процессов, связанных с поиском, добычей и переработкой георесурсов. В вопросах энергосбережения особый интерес вызывают линейные синхронные электромагнитные машины возвратно-поступательного движения ударного и виброударного действия. Частота ударов бойка в данных машинах синхронно связана с частотой источника промышленной сети переменного тока. К главным достоинствам оборудования, использующего линейные синхронные электромагнитные машины ударного и виброударного действия, относится снижение потребления электрической энергии при работе в резонансных режимах или близких к резонансным режимам. Исследование данных режимов связано с построением точных динамических моделей различных модификаций синхронных электромагнитных машин ударного и виброударного действия, работающих исключительно в переходных режимах.

Цель: разработать обобщенную математическую модель двухкатушечной синхронной машины с электромагнитным приводом для технологических систем виброударного действия, упрощающей процесс математического описания известных вариантов схем машин при проведении динамических расчетов.

Методы: диффференциальные уравнения электрического равновесия и механического взаимодействия, поступательно движущихся масс, уравнение Лагранжа второго рода, методы конечно-элементного моделирования двухмерного магнитного поля и структурного моделирования в программе Matlab Simulink, conocтавление расчетных и экспериментальных характеристик.

Результаты. Предложена обобщенная расчетная схема построения многомассовой двухкатушечной синхронной электромагнитной машины, включающая известные модиффикации вариантов схем машин ударного действия. На основе расчетной схемы образована система диффференциальных уравнений обобщенной модели, позволяющая выполнять построение математических моделей, отражающих динамическое состояние известных вариантов схем синхронных ударных машин с электромагнитным приводом. Приведен пример реализации динамической модели четырехмассовой двухкатушечной синхронной электромагнитной машины с упругим реверсом бойка. Приведены результаты имитационного моделирования рабочего режима в виде диаграмм тока катушек, перемещения и скорости бойка, отражающие процессы включения и выхода на квазиустановившийся режим работы. На основе сопоставления значений интегральных характеристик показано, что расхождение результатов расчета и эксперимента составляет $4 . .6 \%$, что не превышает общепринятой погрешности для инженерных расчетов. Эфффективность применения предложенной обобщенной модели заключается в упрощении математического описания вариантов схем синхронных машин с электромагнитным приводом и сокращении сроков реализации моделей.
\end{abstract}

\section{Ключевые слова:}

Электрическая импульсная система, линейная синхронная машина, синхронная частота, число степеней свободы, обобщенная модель, динамическая модель, моделирование.

\section{Введение}

В повышении эффективности интенсификации ряда технологических процессов и производств важную роль играют силовые электрические импульсные системы, способные создавать большие по величине ударные или виброударные воздействия на обрабатываемую среду [1-5].

Высокую долю среди них составляют силовые электромагнитные импульсные системы, содержащие в качестве основного исполнительного механизма ударный или виброударный электропривод на основе различных по конструкции линейных электромагнитных двигателей (ЛЭМД) [6-9]. Генерирование ударных нагрузок в ЛЭМД обеспечивается в результате возвратно-поступательного движения бойка. В самом общем случае подобные структуры, содержащие в своем составе ЛЭМД, устройства для преобразования и передачи механической энергии и рабочий орган импульсной системы, образуют различные модификации электромагнитных машин ударного действия, которые находят все большее применение в прогрессивных технологиях по повышению интенсификации процессов поиска, добычи и переработки георесурсов [10-12].

Перспективным направлением считается применение электромагнитных машин ударного действия в приводах скважинных виброисточников, оказывающих силовое воздействие в обсадной колонне на уровне продуктивного пласта и повышающих интенсификацию процесса добычи нефти из малодебитных скважин $[13,14]$. Их использование существенно повышает эффективность вибросейсмического метода без применения громоздкого наземного оборудования [15]. 
Установлена эффективность применения электромагнитных машин ударного действия различной мощности для зондирующего воздействия на грунт при сейсморазведке полезных ископаемых, что позволяет повысить точность повторения параметров ударных воздействий, упростить производство работ, обеспечить безопасность их проведения для обслуживающего персонала и окружающей среды [16-18].

Обоснована целесообразность использования линейного электропривода на основе электромагнитных машин для гидротехнического строительства морских стационарных платформ, с помощью которых ведется разработка полезных ископаемых на континентальных шельфах [19, 20].

Практика использования мобильных установок на основе электромагнитных машин ударного действия показала свою эффективность в горнодобывающей отрасли при вторичном разрушении горных пород, а также бурении ударным способом шпуров и скважин в твердых породах [21-23].

Значительным результатом следует считать серийное промышленное применение электромагнитных ударных машин в приводах ручного ударного и ударно-вращательного электроинструмента, широко используемого при монтаже в строительстве и в горном деле [24-26].

Развитие и расширение областей применения электромагнитных машин на основе ЛЭМД, прежде всего, связано с вопросами снижения энергопотребления и совершенствования методов их расчета [27, 28].

В вопросах энергосбережения среди известных вариантов схем электромагнитных машин особый интерес представляют синхронные электромагнитные машины ударного или виброударного действия (СЭМУД). В подобных машинах частота возвратнопоступательного движения бойка синхронно связана с частотой питающей промышленной сети переменного тока [29-31]. Основными преимуществами машин и оборудования на основе СЭМУД является возможность снижения потребления электрической энергии от промышленной сети переменного тока при работе в резонансных режимах или режимах близких к резонансным.

В процессе эволюционирования были созданы различные модификации вариантов схем СЭМУД, выполненные в основном по однокатушечным и двухкатушечным вариантам схем.

Разнообразие в вариантах схем, прежде всего, было вызвано необходимостью повышения их надежности, безопасности, улучшением эксплуатационных характеристик при передаче энергии ударом.

Наиболее широкую известность получили модификации вариантов схем двухкатушечных СЭМУД [29].

Существующие варианты схем имеют различную по сложности динамическую систему, способствующую оптимальному процессу формирования импульса силы и передачи его в деформируемую среду.

Сложность расчета подобных динамических систем заключается в прерывистом и многоступенчатом характере преобразования энергии. За счет импульс- ного потребления электрической энергии работа выполняется исключительно в переходных режимах.

В цикличном процессе перемещения бойка преобладают динамические процессы, зависящие от многих факторов, таких как свойства деформируемой среды, связи в механической колебательной системе, свойства применяемых магнитных материалов, сил сопротивления движению бойка и т. д. Точность моделей для решения задач анализа и синтеза в существенной степени будет определяться точностью учета взаимосвязанных факторов.

Важным этапом расчетов является построение точных динамических моделей вариантов схем двухкатушечных СЭМУД. Учитывая их разнообразие, возникает целесообразность в построении обобщенной модели, отражающей динамическое состояние известных модификаций вариантов схем.

Цель исследования состоит в разработке обобщенной математической модели двухкатушечной синхронной машины с электромагнитным приводом для технологических систем виброударного действия, упрощающей процесс математического описания известных вариантов схем машин при проведении динамических расчетов.

\section{Построение обобщенной математической модели двухкатушечной СЭМУД}

В общем случае в механической системе существующих вариантов схем СЭМУД возникают повторяющиеся динамические процессы, которые сопровождаются взаимным преобразованием потенциальной энергии упругих связей и кинетической энергии движущихся инерционных масс с последующей передачей энергии в деформируемую среду. Данные процессы возникают от действия вынуждающих сил электромагнитного поля, периодически возбуждаемого системой катушек.

Расчетная схема построения обобщенной модели многомассовой двухкатушечной СЭМУД, реализующая известные способы возвратно-поступательного движения бойка, представлена на рис. 1. Характер движения ударной массы бойка и управление катушками рабочего - K1 и обратного - K2 хода в обобщенной схеме устанавливается исходя из принятой модификации варианта схемы СЭМУД, согласно с заданным алгоритмом управления.

Расчетная схема (рис. 1) состоит из механической колебательной системы, имеющей пять степеней свободы. Система содержит боёк массой $m_{1}$, который в процессе своего движения периодически взаимодействует с рабочим инструментом массой $m_{2}$ и массивным инерционным преобразователем массой $m_{3}$.

Рабочий инструмент и инерционный преобразователь посредством упругих связей связаны с магнитопроводом и закрепленными внутри катушками с общей массой $m_{4}$. Также механическая колебательная система может содержать дополнительное устройство в форме кольца массой $m_{5}$ для крепления СЭМУД и защиты от вибраций.

Применение дополнительного устройства в сочетании с промежуточным упругим звеном обеспечива- 
ет защиту оператора от вибраций, передаваемых на корпус технологического оборудования. Величина усилия нажатия $-F_{N}$, обеспечивает устойчивый контакт динамической системы с рабочим инструментом при передаче энергии посредством удара при контакте с рабочей поверхностью деформируемой среды.

Работа механической колебательной системы сопровождается постоянными процессами поглощения энергии в упругих связях и потерями энергии за счет возникающих сил трения скольжения между соприкасающимися поверхностями.

Рассматриваемая система имеет пять степеней свободы $(N=5)$. Выбираем в качестве обобщенных переменных координаты линейных перемещений $x_{1} \ldots x_{5}$ центров масс $m_{1} \ldots m_{5}$ (рис. 1$)$.

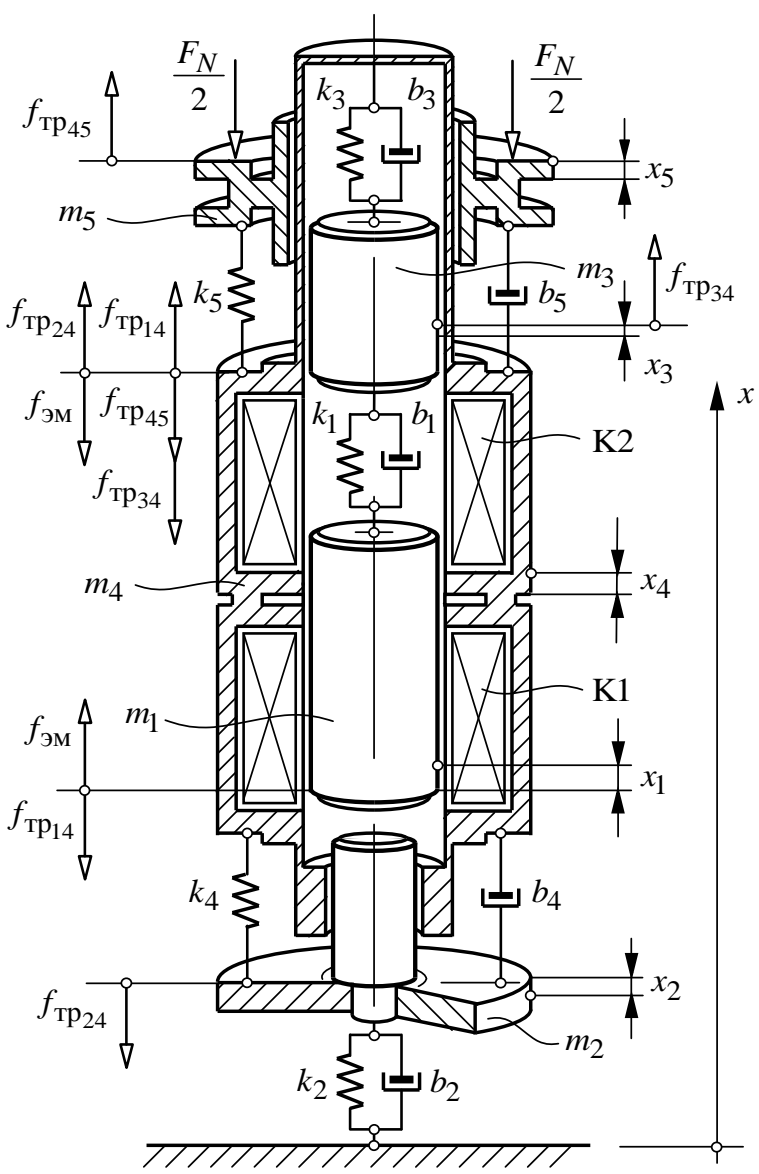

Pис. 1. Расчетная схема обобщенной синхронной машины

Fig. 1. Design diagram of a generalized synchronous machine

При выполнении расчетов полагаем, что массоинерционные элементы механической системы рассматриваются как абсолютно твердые тела, массы которых соответственно равны $m_{1} \ldots m_{5}$. Возникающие в системе ударные взаимодействия рассматриваются как мгновенные изменения скоростей соударяющихся тел. Силы вязкого трения пропорциональны скоростям инерционных масс. Вес бойка значительно меньше сил электромагнитного взаимодействия в механической системе.

В схеме на рис. 1 величина знакопеременной электромагнитной силы

$$
f_{\text {эм }}\left(i_{\mu_{1}}, i_{\mu_{2}}, x_{1}\right)=f_{\text {эм } 1}\left(i_{\mu_{1}}, x_{1}\right)+f_{\text {эм } 2}\left(i_{\mu_{2}}, x_{1}\right)
$$

определяется координатой положения бойка $x_{1}$, а также величиной намагничивающего тока катушки рабочего хода $i_{\mu_{1}}$ и катушки обратного хода $i_{\mu_{2}}$.

Связь электрической и магнитной системы в расчетной схеме может быть учтена с помощью уравнений электромагнитного состояния, предусматривающих насыщение и потери энергии в стальном магнитопроводе на вихревые токи и гистерезис:

$$
\begin{aligned}
& u_{1}(t)=i_{1} r_{1}+\frac{d \psi_{1}\left(i_{\mu_{1}}, x_{1}\right)}{d t}, \\
& u_{2}(t)=i_{2} r_{2}+\frac{d \psi_{2}\left(i_{\mu_{2}}, x_{1}\right)}{d t},
\end{aligned}
$$

где $u_{1}(t), u_{2}(t), i_{1}, i_{1}$ - напряжение и токи в цепях питания катушек; $\psi_{1}=f\left(i_{\mu_{1}}, x_{1}\right), \psi_{2}=f\left(i_{\mu_{2}}, x_{1}\right)$ - потокосцепление катушек; $r_{1}, r_{2}$ - активные сопротивления катушек рабочего и обратного хода.

Общий вид дифференциальных уравнений, описывающих движение центров масс механической системы относительно обобщенных координат, может быть наиболее просто установлен через форму уравнений Лагранжа

$$
\frac{d}{d t}\left(\frac{\partial T}{\partial \dot{x}_{i}}\right)-\frac{\partial T}{\partial x_{i}}=Q_{i}^{\Pi}+Q_{i}^{\Phi}+Q_{i}^{\mathrm{B}},
$$

где $T$ - кинетическая энергия системы; $Q_{i}^{\Pi}, Q_{i}{ }^{\Phi}, Q_{i}^{\mathrm{B}}-$ обобщенные силы, соответствующие потенциальной энергии деформации упругих связей, энергиям рассеяния и внешних воздействий; $x_{i}, \dot{x}_{i}-$ обобщенная координата и обобщенная скорость центров поступательно движущихся масс; $i=1,2,3, \ldots, N$ - число степеней свободы системы.

Для построения модели динамического состояния СЭМУД необходимо, обращаясь к расчетной схеме на рис. 1, выполнить операции дифференцирования левой части уравнения (3) и определить обобщенные силы системы, входящие в правую часть этого уравнения.

Рассматривая поступательные движения масс, кинетическая энергия системы

$$
\begin{gathered}
T=\frac{1}{2} \sum_{i=1}^{N=5} m_{i} \dot{x}_{i}^{2}= \\
=\frac{1}{2}\left[m_{1} \dot{x}_{1}^{2}+m_{2} \dot{x}_{2}^{2}+m_{3} \dot{x}_{3}^{2}+m_{4} \dot{x}_{4}^{2}+m_{5} \dot{x}_{5}^{2}\right] .
\end{gathered}
$$

Обобщенные силы, вызванные деформацией упругих связей

$$
\begin{aligned}
& Q_{i}^{\Pi}=-\frac{\partial \Pi}{\partial x_{i}}=-\frac{\partial}{\partial x_{i}} \sum_{i=1}^{N=5} \frac{1}{2}\left[k_{1}\left(x_{1}-x_{3}\right)^{2}+k_{2} x_{2}^{2}+\right. \\
& \left.+k_{3}\left(x_{4}-x_{3}\right)^{2}+k_{4}\left(x_{4}-x_{2}\right)^{2}+k_{5}\left(x_{5}-x_{4}\right)^{2}\right]
\end{aligned}
$$

где П - потенциальная энергия; $k_{1} \ldots k_{5}$ - коэффициенты жесткости упругих связей.

Обобщенные силы, соответствующие энергии рассеивания 


$$
\begin{gathered}
Q_{i}^{\Phi}=-\frac{\partial \Phi}{\partial \dot{x}_{i}}=-\frac{\partial}{\partial \dot{x}_{i}} \sum_{i=1}^{N=5} \frac{1}{2}\left[b_{1}\left(\dot{x}_{1}-\dot{x}_{3}\right)^{2}+b_{2} \dot{x}_{2}^{2}+\right. \\
\left.+b_{3}\left(\dot{x}_{4}-\dot{x}_{3}\right)^{2}+b_{4}\left(\dot{x}_{4}-\dot{x}_{2}\right)^{2}+b_{5}\left(\dot{x}_{5}-\dot{x}_{4}\right)^{2}\right],
\end{gathered}
$$

где $\Phi$ - диссипативная функция (функция Рэлея); $b_{1} \ldots b_{5}-$ коэффициенты линейного сопротивления, характеризующие диссипативные свойства упругих связей (вязкое трение).

Проекции обобщенной силы, вызванные трением скольжения и внешними воздействиями,

$$
\left\{\begin{array}{l}
Q_{x_{1}}^{\mathrm{B}}=f_{\text {эм }}\left(i_{\mu_{1}}, i_{\mu_{2}}, x_{1}\right)-f_{\mathrm{Tp}_{14}} \operatorname{sign} \frac{d x_{1}}{d t} \\
Q_{x_{2}}^{\mathrm{B}}=-f_{\mathrm{Tp}_{24}} \operatorname{sign} \frac{d x_{2}}{d t} ; \\
Q_{x_{3}}^{\mathrm{B}}=f_{\mathrm{Tр}_{34}} \operatorname{sign} \frac{d x_{3}}{d t} ; \\
Q_{x_{4}}^{\mathrm{B}}=-f_{\text {эм }}\left(i_{\mu_{1}}, i_{\mu_{2}}, x_{1}\right)+ \\
+\left(-f_{\mathrm{Tp}_{34}}-f_{\mathrm{Tp}_{45}}+f_{\mathrm{Tp}_{24}}+f_{\mathrm{Tp}_{14}}\right) \operatorname{sign} \frac{d x_{4}}{d t} ; \\
Q_{x_{5}}^{\mathrm{B}}=f_{\mathrm{Tp}_{45}} \operatorname{sign} \frac{d x_{5}}{d t}-F_{N},
\end{array}\right.
$$

где $f_{\mathrm{Tp}_{14}}, f_{\mathrm{Tp}_{24}}, f_{\mathrm{Tp}_{34}}, f_{\mathrm{Tp}_{45}}-$ силы сухого трения скольжения в соответствии с рис. 1 .

Выражения (4)-(7) совместно с (3) позволяют образовать уравнения динамического состояния механической колебательной системы в виде следующих дифференциальных уравнений:

$$
\begin{gathered}
m_{1} \frac{d^{2} x_{1}}{d t^{2}}+b_{1}\left(\frac{d x_{1}}{d t}-\frac{d x_{3}}{d t}\right)+k_{1}\left(x_{1}-x_{3}\right)= \\
=f_{\text {эм }}\left(i_{\mu_{1}}, i_{\mu_{2}}, x_{1}\right)-f_{\mathrm{Tp}_{14}} \operatorname{sign} \frac{d x_{1}}{d t} ; \\
m_{2} \frac{d^{2} x_{2}}{d t^{2}}+b_{2} \frac{d x_{2}}{d t}-b_{4}\left(\frac{d x_{4}}{d t}-\frac{d x_{2}}{d t}\right)+k_{2} x_{2}- \\
-k_{4}\left(x_{4}-x_{2}\right)=-f_{\mathrm{Tp}_{24}} \operatorname{sign} \frac{d x_{2}}{d t} ; \\
m_{3} \frac{d^{2} x_{3}}{d t^{2}}-b_{1}\left(\frac{d x_{1}}{d t}-\frac{d x_{3}}{d t}\right)-b_{3}\left(\frac{d x_{4}}{d t}-\frac{d x_{3}}{d t}\right)- \\
-k_{1}\left(x_{1}-x_{3}\right)-k_{3}\left(x_{4}-x_{3}\right)=f_{\mathrm{Tp}_{34}} \operatorname{sign} \frac{d x_{3}}{d t} ; \\
m_{4} \frac{d^{2} x_{4}}{d t^{2}}+b_{3}\left(\frac{d x_{4}}{d t}-\frac{d x_{3}}{d t}\right)+b_{4}\left(\frac{d x_{4}}{d t}-\frac{d x_{2}}{d t}\right)- \\
-b_{5}\left(\frac{d x_{5}}{d t}-\frac{d x_{4}}{d t}\right)+k_{3}\left(x_{4}-x_{3}\right)+k_{4}\left(x_{4}-x_{2}\right)- \\
-k_{5}\left(x_{5}-x_{4}\right)=-f_{\text {эм }}\left(i_{\mu_{1}}, i_{\mu_{2}}, x_{1}\right)+ \\
\left.+f_{\mathrm{Tp}_{45}}+f_{\text {тр}_{24}}+f_{\mathrm{Tp}_{14}}\right) \operatorname{sign} \frac{d x_{4}}{d t} ;
\end{gathered}
$$

$$
\begin{gathered}
m_{5} \frac{d^{2} x_{5}}{d t^{2}}+b_{5}\left(\frac{d x_{5}}{d t}-\frac{d x_{4}}{d t}\right)+k_{5}\left(x_{5}-x_{4}\right)= \\
=f_{\mathrm{Tp}_{45}} \operatorname{sign} \frac{d x_{5}}{d t}-F_{N} .
\end{gathered}
$$

Уравнения (8)-(12) рассматривают только колебательные свойства механической системы с потерями энергии.

Дополняя уравнения электрического равновесия $(1),(2)$ и динамического состояния механической системы (8)-(12) условиями, ограничивающими пределы возвратно-поступательного движения бойка, на границах которых возникают ударные взаимодействия, переходим к окончательному процессу формирования динамической модели с учетом рассматриваемой модификации двухкатушечной СЭМУД.

Полученную модель можно использовать для анализа рабочих процессов СЭМУД в переходных и квазиустановившихся режимах.

Процесс решения уравнений относительно принятого конструктивного исполнения СЭМУД должен сопровождаться предварительным расчетом функциональных зависимостей потокосцепления и электромагнитной силы $\psi_{1}=f\left(i_{\mu_{1}}, x_{1}\right), \psi_{2}=f\left(i_{\mu_{2}}, x_{1}\right), f_{\text {эм } 1}^{\prime}=f\left(i_{\mu_{1}}, x_{1}\right)$, $f_{\text {эм} 2}^{\prime}=f\left(i_{\mu_{2}}, x_{1}\right)$ от координаты смещения бойка и намагничивающего тока катушек. Значения указанных функциональных зависимостей в процессе моделирования определяются с помощью интерполяции табличных данных, полученных в результате решения полевой задачи в статике для различных комбинаций положения бойка и протекающего по катушкам намагничивающего тока с использованием современных методов численного расчета двухмерного магнитного поля [32-36].

\section{Реализация динамической модели на примере} двухкатушечной СЭМУД с упругим реверсом бойка

Основные этапы использования обобщенной модели рассмотрим на примере реализации динамической модели четырехмассовой двухкатушечной СЭМУД с упругим реверсом бойка, представленной на рис. 2.

Впервые подобный вариант схемы был применен в конструкции ударного узла магнитобура СЦ-2 для бурения ударно-вращательным способом отверстий в бетоне и твердых породах, а также в ударных узлах буровых установок для разрушения горных пород [37].

Вариант схемы СЭМУД (рис. 2) содержит боек - 1 , катушку рабочего хода -2 и катушку обратного хода - 3. Обе катушки размещены внутри общего магнитопровода - 4, выполненного из листов электротехнической стали. При протекании тока по катушкам создается магнитное поле, с которым взаимодействует боек и совершает возвратно-поступательные движения. В процессе колебательных движений боек - 1 поочередно взаимодействует с упругим звеном - 5, выполненным в виде цилиндрической пружины, работающей на сжатие, и с торцевой поверхностью рабочего инструмента - 6. В результате этих 
взаимодействий генерируется ударный импульс силы, передаваемый далее в деформируемую среду для совершения полезной работы.

Для гашения вертикальных вибраций применяется виброизолирующее устройство, состоящее из промежуточной массы - 7 и упругого звена, выполненного в виде цилиндрической пружины - 8, работающей на сжатие. Для устойчивой связи рабочего инструмента с деформируемой средой прилагается внешняя статическая сила нажатия $F_{N}$, передаваемая через промежуточную массу инструменту. Питание катушек рабочего и обратного хода выполняется импульсами тока по однополупериодной схеме выпрямления.

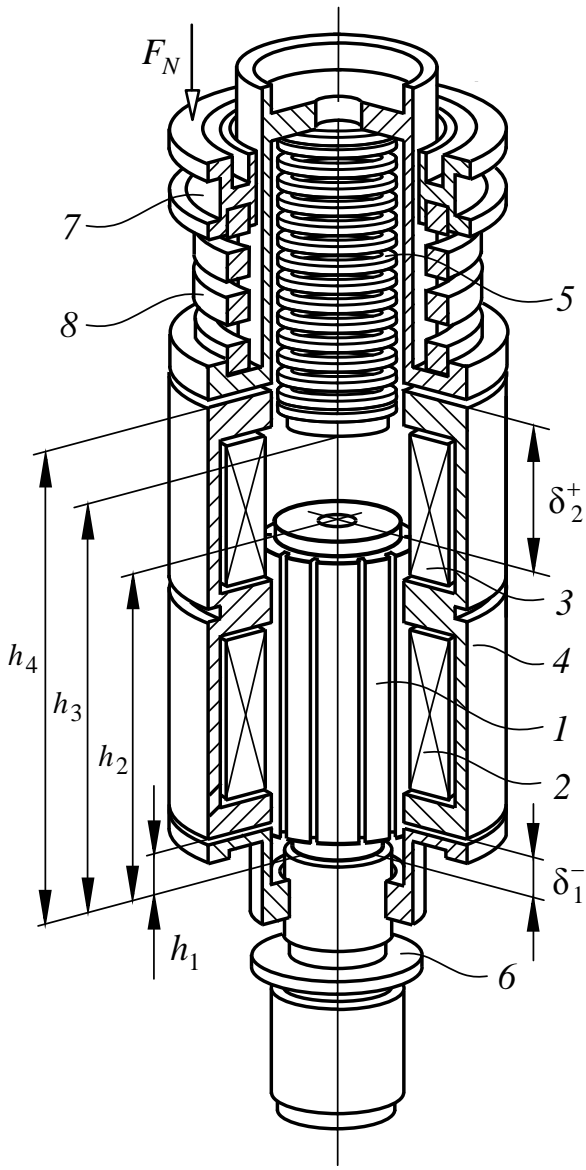

Рис. 2. Вариант схемьг двухкатушечной синхронной машины

Fig. 2. Variant of the scheme of a two-coil synchronous machine

За промежуток времени, равный одному периоду напряжения промышленной сети, осуществляется полный рабочий цикл машины. Для частоты сети $f=50$ Гц количество ударов бойка составляет $n_{\text {уд }}=3000$ уд/мин.

Рассматривая механическую колебательную систему рис. 2 и сопоставляя движение инерционных масс с расчетной схемой на рис. 1 , следует полагать, что для реализации динамической модели следует принять: $m_{3}=0, k_{3}=0, b_{3}=0, x_{3}=x_{4}, \dot{x}_{3}=\dot{x}_{4}, f_{\text {тр } 34}=0, N=4$.

Предварительно объединив на основе равенства обобщенных координат $x_{3}=x_{4}$ левые и правые части уравнений (10), (11), а также произведя некоторую замену, получим уравнения, описывающие динамическое состояние четырехмассовой механической колебательной системы по варианту схемы на рис. 2:

$$
\begin{gathered}
m_{1} \frac{d^{2} x_{1}}{d t^{2}}+b_{1}\left(\frac{d x_{1}}{d t}-\frac{d x_{4}}{d t}\right)+k_{1}\left(x_{1}-x_{4}\right)= \\
=f_{\text {эм }}\left(i_{\mu_{1}}, i_{\mu_{2}}, x_{1}\right)-f_{\mathrm{Tp}_{14}} \operatorname{sign} \frac{d x_{1}}{d t} ; \\
m_{2} \frac{d^{2} x_{2}}{d t^{2}}+b_{2} \frac{d x_{2}}{d t}-b_{4}\left(\frac{d x_{4}}{d t}-\frac{d x_{2}}{d t}\right)+ \\
+k_{2} x_{2}-k_{4}\left(x_{4}-x_{2}\right)=-f_{\mathrm{Tp}_{24}} \operatorname{sign} \frac{d x_{2}}{d t} ; \\
m_{4} \frac{d^{2} x_{4}}{d t^{2}}-b_{1}\left(\frac{d x_{1}}{d t}-\frac{d x_{4}}{d t}\right)+b_{4}\left(\frac{d x_{4}}{d t}-\frac{d x_{2}}{d t}\right)- \\
-b_{5}\left(\frac{d x_{5}}{d t}-\frac{d x_{4}}{d t}\right)-k_{1}\left(x_{1}-x_{4}\right)+k_{4}\left(x_{4}-x_{2}\right)- \\
-k_{5}\left(x_{5}-x_{4}\right)=-f_{\text {эм }}\left(i_{\mu_{1}}, i_{\mu_{2}}, x_{1}\right)+ \\
+\left(f_{\mathrm{тр}_{45}}+f_{\mathrm{тр}_{24}}+f_{\mathrm{тр}_{14}}\right) \operatorname{sign} \frac{d x_{4}}{d t} ; \\
m_{5} \frac{d^{2} x_{5}}{d t^{2}}+b_{5}\left(\frac{d x_{5}}{d t}-\frac{d x_{4}}{d t}\right)+k_{5}\left(x_{5}-x_{4}\right)= \\
\operatorname{sign} \frac{d x_{5}}{d t}-F_{N} \cdot
\end{gathered}
$$

Окончательный результат движения бойка необходимо рассматривать как процесс совместного действия вынуждающих электромагнитных сил и ударных импульсов сил, возникающих вследствие периодического взаимодействия бойка с рабочим инструментом.

В качестве ограничительных условий здесь выступают границы пределов поступательного перемещения бойка, гарантирующие его торможение, остановку и реверс в заданных границах. Данные пределы целесообразно рассматривать относительно промежутков рабочих воздушных зазоров $\delta_{1}, \delta_{2}$ либо введенных фиксированных промежутков, определяемых расстояниями до ударного сечения $h_{1}, h_{2}, h_{3}$ и $h_{4}$ (рис. 2).

Ограничения, вызванные торможением и остановкой бойка согласно рис. 2, могут быть установлены в виде следующей системы равенств:

$$
x_{1}(t)=\left\{\begin{array}{l}
0, \text { при } \delta_{2}^{+}=h_{4}-h_{2} ; \\
h_{4}-h_{2}-\delta_{2}^{+}, \quad \text { при } 0 \leq x_{1} \leq h_{4}-h_{2} ; \\
h_{4}-h_{2}+\left|\delta_{2}^{-}\right|, \text {при } x_{1}>h_{4}-h_{2},
\end{array}\right.
$$

Также важным условием для реализации модели является связь между кинетической энергией бойка, направленной на совершение полезной работы $T_{1}$ и возвращаемой обратно в механическую систему $T_{2}$.

Связь этой энергии можно установить через соотношение скоростей ударной массы бойка до удара $v_{1}$ и после удара $\bar{v}_{1}$ в виде: 


$$
T_{1}=\frac{m_{1} v_{1}^{2}}{2}\left(1-k_{\mathrm{oT}}^{2}\right) ; T_{2}=\frac{m_{1} v_{1}^{2}}{2} k_{\mathrm{oT}}^{2},
$$

где $k_{\text {от }}=\bar{v}_{1 /} v_{1}-$ коэффициент отскока.

Работа упругого звена при взаимодействии с бойком может быть описана следующей системой равенств, содержащих кусочно-линейные функции (рис. 2):

$$
\begin{aligned}
& \frac{\partial \Pi}{d x_{1}}=\left\{\begin{array}{l}
0, \quad \text { при } 0 \leq x_{1}<h_{3}-h_{2} ; \\
k_{1}\left(x_{1}-x_{4}\right), \quad \text { при } x_{1} \geq h_{3}-h_{2},
\end{array}\right. \\
& \frac{\partial \Phi}{d \dot{x}_{1}}=\left\{\begin{array}{l}
0, \text { при } 0 \leq x_{1}<h_{3}-h_{2} ; \\
b_{1}\left(\frac{d x_{1}}{d t}-\frac{d x_{4}}{d t}\right), \quad \text { при } x_{1} \geq h_{3}-h_{2},
\end{array}\right.
\end{aligned}
$$

Окончательно принимая во внимание уравнения (1), (2), (13)-(16), с учетом установленных условий и ограничений (17)-(20), математическая модель варианта схемы СЭМУД на рис. 2 может быть представлена в виде системы:

$$
\left\{\begin{array}{l}
u_{1}(t)=i_{1} r_{1}+\frac{d \psi_{1}\left(i_{\mu 1}, x_{1}\right)}{d t} \\
u_{2}(t)=i_{2} r_{2}+\frac{d \psi_{2}\left(i_{\mu 2}, x_{1}\right)}{d t} \\
i_{1}=i_{\mathrm{n} 1}+i_{\mu 1} ; \quad i_{2}=i_{\mathrm{n} 2}+i_{\mu 2} \\
m_{1} \frac{d^{2} x_{1}}{d t^{2}}=A \\
m_{2} \frac{d^{2} x_{2}}{d t^{2}}=B \\
m_{4} \frac{d^{2} x_{4}}{d t^{2}}=C \\
m_{5} \frac{d^{2} x_{5}}{d t^{2}}=D
\end{array}\right.
$$

Установленные в системе (21) переменные коэффициенты:

$$
A=\left\{\begin{array}{l}
\frac{m_{1} \bar{v}_{1}}{\tau_{\text {и }}}+f_{\text {эм }}\left(i_{\mu_{1}}, i_{\mu_{2}}, x_{1}\right)-f_{\text {тр}_{14}} \operatorname{sign} \frac{d x_{1}}{d t}, \\
\text { если } \frac{d x_{1}}{d t}>0, \quad \text { при } 0 \leq x_{1}<h_{3}-h_{2} ; \\
-\tilde{\lambda}_{1}+f_{\text {эм }}\left(i_{\mu_{1}}, i_{\mu_{2}}, x_{1}\right)-f_{\text {тр}_{14}} \operatorname{sign} \frac{d x_{1}}{d t}, \\
\text { если } \frac{d x_{1}}{d t}>0, \text { при } x_{1} \geq h_{3}-h_{2} ; \\
f_{\text {эм }}\left(i_{\mu_{1}}, i_{\mu_{2}}, x_{1}\right)-f_{\text {тр}_{14}} \operatorname{sign} \frac{d x_{1}}{d t}, \\
\text { если } \frac{d x_{1}}{d t}<0, \text { при } 0 \leq x_{1}<h_{3}-h_{2} ; \\
B=-\frac{m_{2} \bar{v}_{2}}{\tau_{\text {и }}}-\tilde{\lambda}_{2}+\tilde{\lambda}_{4}-f_{\text {тр }_{24}} \operatorname{sign} \frac{d x_{2}}{d t} ;
\end{array}\right.
$$

$$
\begin{aligned}
& C=\left\{\begin{array}{l}
-\tilde{\lambda}_{3}+\tilde{\lambda}_{5}-f_{\text {эм }}\left(i_{\mu_{1}}, i_{\mu_{2}}, x_{1}\right)+ \\
+\left(f_{\mathrm{Tp}_{14}}-f_{\mathrm{тр}_{24}}\right) \operatorname{sign} \frac{d x_{4}}{d t}, \\
\text { при } 0 \leq x_{1}<h_{3}-h_{2} ; \\
\tilde{\lambda}_{1}-\tilde{\lambda}_{3}-f_{\text {эм }}\left(i_{\mu_{1}}, i_{\mu_{2}}, x_{1}\right)- \\
-\left(f_{\mathrm{Tp}_{14}}-f_{\mathrm{Tр}_{24}}+f_{\mathrm{тp}_{45}}\right) \operatorname{sign} \frac{d x_{4}}{d t}, \\
\text { при } x_{1} \geq h_{3}-h_{2} ;
\end{array}\right. \\
& D=-\tilde{\lambda}_{5}+f_{\mathrm{Tp}_{45}} \operatorname{sign} \frac{d x_{5}}{d t}-F_{N},
\end{aligned}
$$

где

$$
\begin{gathered}
\tilde{\lambda}_{1}=k_{1}\left(x_{1}-x_{4}\right)+b_{1}\left(\frac{d x_{1}}{d t}-\frac{d x_{4}}{d t}\right) ; \tilde{\lambda}_{2}=k_{2} x_{2}+b_{2} \frac{d x_{2}}{d t} ; \\
\tilde{\lambda}_{4}=k_{4}\left(x_{4}-x_{2}\right)+b_{4}\left(\frac{d x_{4}}{d t}-\frac{d x_{2}}{d t}\right) ; \\
\tilde{\lambda}_{5}=k_{5}\left(x_{5}-x_{4}\right)+b_{5}\left(\frac{d x_{5}}{d t}-\frac{d x_{4}}{d t}\right) ;
\end{gathered}
$$

$m_{1} \bar{v}_{1}, m_{2} \bar{v}_{2}-$ импульсы сил при ударе; $\tau_{\text {и }}-$ время действия импульса; $\bar{v}_{2}$ - скорость приобретенная рабочим инструментом; $i_{\Pi_{1}}, i_{\Pi_{2}}$ - токи потерь, обусловленные процессами в стальном магнитопроводе.

Полученная модель в виде системы дифференциальных уравнений (21) позволяет производить всесторонний анализ динамических процессов, происходящих в двухкатушечном СЭМУД с упругим реверсом бойка.

Решение системы (21) выполнялось в соответствии с существующими рекомендациями [38, 39] в программе Matlab Simulink.

При выполнении динамических расчетов использовались параметры модернизированного промышленного варианта СЭМУД «ИЭ-4207»: $m_{1}=0,394$ кг; $m_{2}=0,32$ кг; $m_{4}=3,9$ кг; $m_{5}=0,2$ кг $k_{1}=120 \cdot 10^{3} \mathrm{H} / \mathrm{M}$; $b_{1}=8 \mathrm{H} \cdot \mathrm{c} / \mathrm{M} ; \quad k_{2}=12 \cdot 10^{6} \mathrm{H} / \mathrm{M} ; \quad b_{2}=0 ; \quad k_{4}=16 \cdot 10^{3} \mathrm{H} / \mathrm{M}$; $b_{4}=35 \mathrm{H} \cdot \mathrm{c} / \mathrm{M} ; f_{\text {тр } 14}=3 \mathrm{H} ; f_{\text {тр } 24}=8 \mathrm{H} ; f_{\text {тр } 45}=5 \mathrm{H} ; F_{N}=35 \mathrm{H}$.

Для уменьшения потерь магнитопровод выполнен в виде пакетов из электротехнической стали 1212 толщиной 0,35 мм. Боек цельный из стали 40ХC.

Катушки выполнены медным проводником круглого сечения с использованием каркасной намотки. Параметры катушки рабочего хода: $w_{1}=1290, r_{1}=12,9$ Ом. Параметры катушки обратного хода: $w_{2}=1540, r_{2}=23,2$ Ом. Обе катушки независимо включены на переменное напряжение $U=220$ В через неуправляемые вентили и поочередно питаются от полуволн напряжения разной полярности.

Предварительный расчет функциональных зависимостей потокосцеплений и электромагнитных сил $\psi_{1}=f\left(i_{1}, \delta_{1}\right), \psi_{2}=f\left(i_{2}, \delta_{2}\right), f_{\text {эм } 1}^{\prime}=f\left(i_{1}, \delta_{1}\right), f_{\text {эм } 2}^{\prime}=f\left(i_{2}, \delta_{2}\right)$ от величины воздушного рабочего зазора и тока катушек выполнялся в программе FEMM (Finite Element Method Magnetics) [40]. 
Полученные по результатам полевого расчета статических параметров трехмерные графики зависимости $\psi_{1}=f\left(i_{1}, \delta_{1}\right)$ и $f_{\text {эм1 }}=f\left(i_{1}, \delta_{1}\right)$ для катушки рабочего хода, содержащие по 342 опорные точки статических параметров, представлены на рис. 3,4 .

Расчет составляющих токов $i_{\text {п1 }}$ и $i_{\text {п2 }}$ выполнялся по методике, изложенной в работе [41].

Результаты имитационного моделирования в виде диаграмм тока катушек, перемещения и скорости бойка, отражающие процесс включения СЭМУД и выхода на квазиустановившийся режим работы, рассмотрены на рис. 5-8.

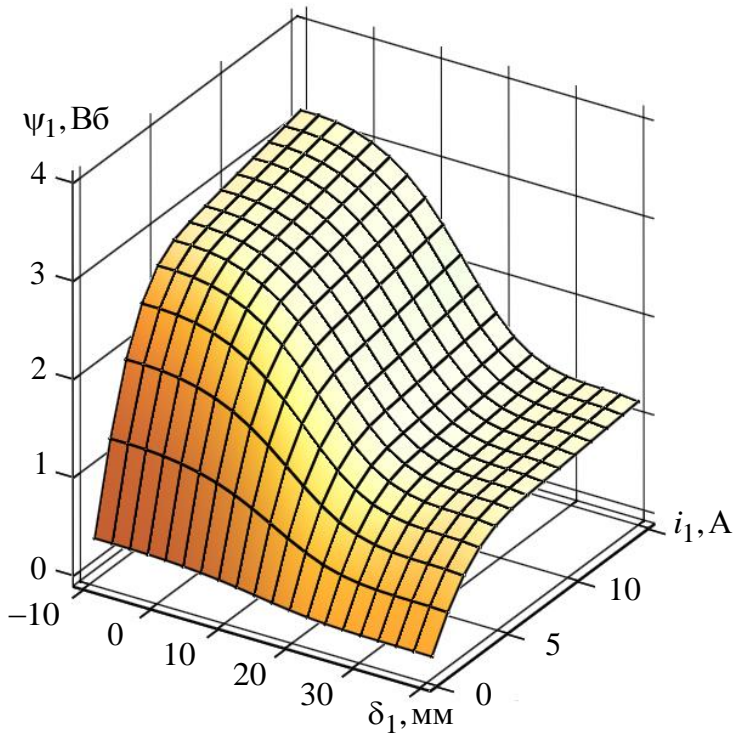

Рис. 3. Трехмерный график расчета потокосиепления $\psi_{1}=f\left(i_{1}, \delta_{l}\right)$

Fig. 3. Three-dimensional graph for calculating flux linkage $\psi_{1}=f\left(i_{1}, \delta_{l}\right)$

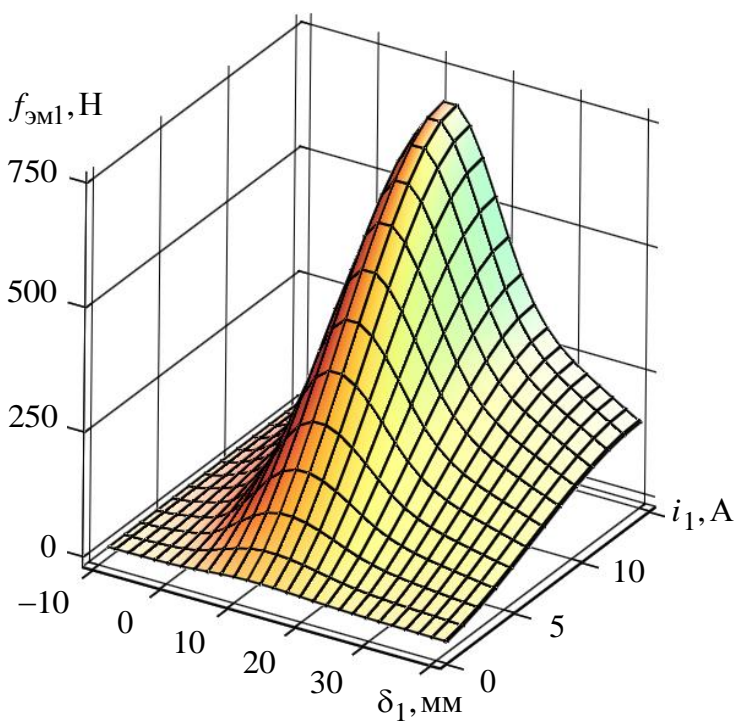

Рис. 4. Трехмерный график расчета электромагнитного усилия $f_{\text {эмI }}=f\left(i_{1}, \delta_{1}\right)$

Fig. 4. Three-dimensional graph for calculating the electromagnetic force $f_{\text {eml }}=f\left(i_{1}, \delta_{1}\right)$
Диаграммы характеризуются устойчивым периодическим режимом работы двухкатушечной СЭМУД с начальной скоростью при отскоке бойка $\bar{v}_{1}=0,5 \mathrm{~m} / \mathrm{c}$ и коэффициентом отскока $k_{\text {ог }}=0,11$.

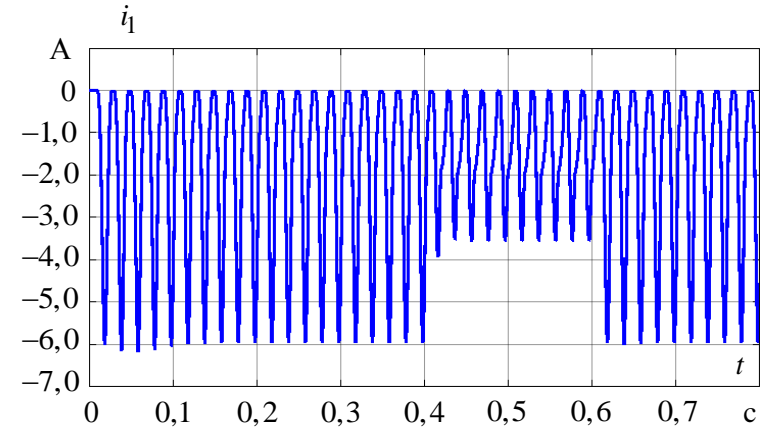

Рис. 5. Диаграмма тока $i_{l}(t)$ катушки рабочего хода

Fig. 5. Current diagram of the travel coil $i_{l}(t)$

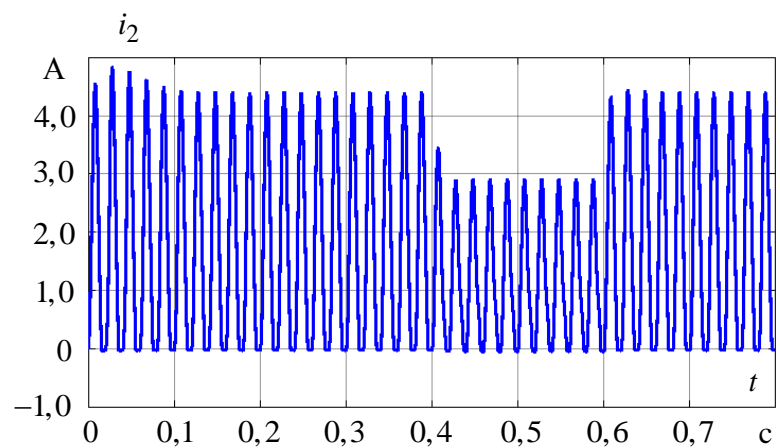

Рис. 6. Диаграмма тока $i_{2}(t)$ катушки обратного хода

Fig. 6. Current diagram of the reverse coil $i_{2}(t)$

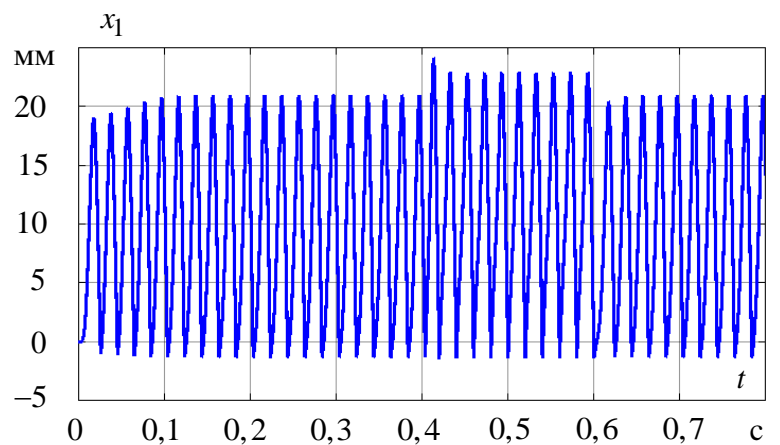

Pис. 7. Диаграмма перемещения бойка $x_{I}(t)$

Fig. 7. Movement diagram of the striker $x_{I}(t)$

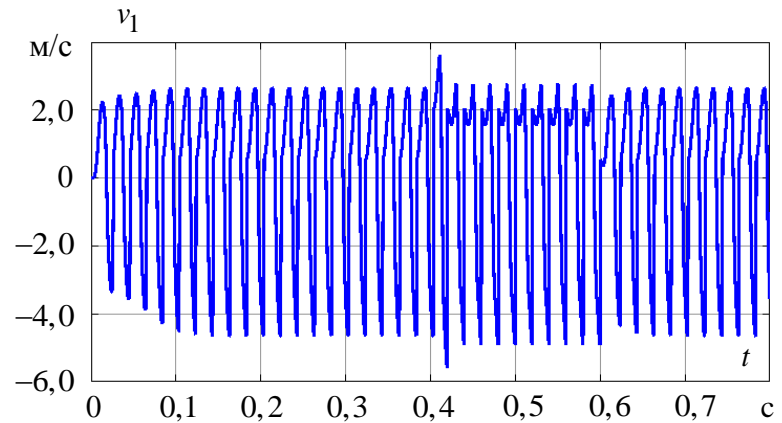

Pис. 8. Диаграмма скорости бойка $v_{l}(t)$

Fig. 8. Striker speed chart $v_{l}(t)$ 
Также на рис. 5-8 отражена реакция модели СЭМУД по восстановлению колебательных свойств вследствие внезапного изменения упругих свойств деформируемой среды на интервале времени $t=0,4 \ldots 0,6 \mathrm{c}$

С увеличением упругих свойств деформируемой среды на указанном интервале времени увеличивается начальная скорость отскока бойка $\bar{v}_{1}=2,0$ м/с и коэффициент отскока $k_{\text {от }}=0,43$. Заметно повышается амплитуда колебания бойка (рис. 7) при сохранении устойчивого режима работы модели. Максимальная скорость перемещения бойка (рис. 8) практически не изменяется.

Также наблюдается уменьшение амплитуды тока катушки рабочего хода (рис. 5) и катушки обратного хода (рис. 6), что вызывает снижение мощности, потребляемой от источника питания. Данное уменьшение объясняется тем, что с увеличением коэффициента отскока возрастает кинетическая энергия, возвращаемая в механическую систему при обратном ходе бойка. В то время как кинетическая энергия, передаваемая в деформируемую среду, снижается.

Таким образом, частичное снижение полезной мощности и мощности, потребляемой от источника, не нарушают колебательных свойств механической системы. Рабочий процесс характеризуются устойчивым режимом работы без применения специальных средств регулирования.

Таблица. Сравнение результатов

Table. Comparison of results

\begin{tabular}{|l|c|c|c|c|c|}
\hline $\begin{array}{c}\text { Интегральный } \\
\text { показатель } \\
\text { Integral indicator }\end{array}$ & $\begin{array}{c}A_{\text {im, }, \text { Дж }} \\
\text { (J) }\end{array}$ & $\begin{array}{c}I, A \\
\text { (a) }\end{array}$ & $\begin{array}{c}P_{1}, \mathrm{Bт} \\
\text { (W) }\end{array}$ & $\begin{array}{c}P_{2}, \text { Вт } \\
\text { (W) }\end{array}$ & $\begin{array}{c}\text { КПД } \\
\text { Efficiency, } \\
\%\end{array}$ \\
\hline $\begin{array}{l}\text { Pacчет } \\
\text { Calculation }\end{array}$ & 4,21 & 3,54 & 492 & 211 & 41 \\
\hline $\begin{array}{l}\text { Эксперимент } \\
\text { Experiment }\end{array}$ & 4,18 & 3,6 & 536 & 209 & 39 \\
\hline
\end{tabular}

Оценивание точности построения модели выполнялось сравнением интегральных значений показателей энергии удара $-A_{\text {in }}$, действующего значения тока на входе $-I$, активной мощности $-P_{1}$, полезной мощности на выходе машины $-P_{2}$ и КПД, полученных в ходе численного расчета с показателями, найденными с помощью физической модели СЭМУД с упругим реверсом бойка (рис. 2). Сравнение результатов рас-

\section{СПИСОК ЛИТЕРАТУРЫ}

1. Ашавский А.М., Вольперт А.Я., Шнейнбаум В.С. Силовые импульсные системы. - М.: Машиностроение, 1978. -200 с.

2. Абрамов А.Д., Тюнюкова Т.К., Ижбулдин Е.А. Виброударные технологии для строительно-дорожных машин // Мир транспорта. - 2016. - Т. 14. - № 4 (65). - С. 62-69.

3. Абрамов А.Д., Галай М.С. Перспективы применения машин ударного действия при упрочнении сварных швов // Фундаментальные и прикладные вопросы горных наук. - 2016. - Т. 2. № 3. - C. 7-10.

4. Moshkin V.I. Pulsing linear electromagnetic motor mechanical work within the desaturated magnetic system // International Conference on Industrial Engineering, Applications and Manufacturing, ICIEAM, 2017. - P. 8076319.

5. Нейман В.Ю. Интегрированные линейные электромагнитные двигатели для импульсных технологий // Электротехника. 2003. - № 9. - C. 25-30. чета и физического эксперимента представлено в таблице.

Расхождение результатов расчета (таблица) с результатами, полученными с помощью физической модели модернизированного варианта схемы СЭМУД с упругим реверсом бойка, составляет $4 . .6 \%$.

По результатам моделирования установлено, что точность в определении выходных характеристик моделей при выполнении динамических расчетов в значительной степени определяется точностью установки входных параметров моделей. В особенности это относится к точности выбора числа степеней свободы механической системы, обобщенных сил, связанных с энергией рассеивания, а также сил, вызванных трением скольжения и внешними воздействиями. Как правило, это параметры на входе модели, которыми разработчики чаще всего пренебрегают или пытаются существенно их упростить.

\section{Заключение}

Предложена обобщенная расчетная схема построения многомассовой двухкатушечной СЭМУД, включающая известные варианты схем машин.

На основе расчетной схемы образована система дифференциальных уравнений, позволяющая при установленных ограничениях относительно существующих модификаций вариантов схем выполнять построение математических моделей двухкатушечных СЭМУД, характеризующихся многосторонними связями в механической системе, существенной нелинейностью электромагнитных тяговых характеристик и потокосцеплений, а также наличием ударной нагрузки и потерями энергии в электрической, магнитной и механической системе.

На примере четырехмассовой конструкции двухкатушечной СЭМУД с упругим реверсом бойка рассмотрен пример реализации математической модели для анализа динамических характеристик. Расхождение расчетных значений интегральных показателей с экспериментальными данными, полученными на физической модели, не превышает погрешности 4...6\%, допустимой для инженерных расчетов.

Эффективность предложенной обобщенной модели заключается в упрощении процесса математического описания вариантов схем СЭМУД при сокращении сроков реализации моделей.

6. Design and analysis of a new down-the-hole electromagnetic hammer driven by tube linear motor / T. Wu, Y. Tang, S. Tang, Y. Li, W. He, E. Chen // IET Electric Power Applications. 2017. - V. 11 (9). - P. 1558-1565.

7. Electric converters of electromagnetic strike machine with battery power / K.M. Usanov, A.V. Volgin, V.A. Kargin, A.P. Moiseev, E.A. Chetverikov // IOP Conference Series: Materials Science and Engineering. - 2018. - V. 327. - P. 052032.

8. Effect of vibroimpact treatment on the physical and mechanical properties of the surface layer of welded joints in rails / V.A. Kargin, L.B. Tikhomirova, A.D. Abramov, M.S. Galai // Welding International. - 2014. - V. 28 (3). - P. 245-247.

9. Neyman L.A., Neyman V.Yu., Shabanov A.S. Vibration dynamics of an electromagnetic drive with a half-period rectifier // The 18 international conference of young specialists on micro/nanotechnologies and electron devices: Proc. - Altai, Erlagol, 29 June -3 July 2017. - P. 503-506. 
10. Yedygenov E.K., Lyashkov V. Electromagnetic rock breaker for non-explosive rocks breaking // International Symposium on planning of mining and selection of equipment of MPES 2011. Almata, 2011. - P. 1002-1012

11. Павлов В.Е. Исследование режимов работы электромагнитного молота // Вестник Иркутского государственного технического университета. - 2017. - Т. 21. - № 12 (131). - С. 164-173.

12. Угаров Г.Г., Нейман В.Ю. Анализ показателей электромагнитных ударных машин // Физико-технические проблемы разработки полезных ископаемых. - 1996. - № 2. - С. 72-80.

13. Экспериментальные исследования эффективности излучения от скважинного импульсного виброисточника / Б.Ф. Симонов, В.Н. Опарин, А.О. Кордубайло, В.И. Востриков // Горный информационно-аналитический бюллетень (научно-технический журнал). - 2019. - № 8. - С. 180-189.

14. Симонов Б.Ф., Нейман В.Ю., Шабанов А.С. Импульсный линейный электромагнитный привод для скважинного виброисточника // Физико-технические проблемы разработки полезных ископаемых. - 2017. - № 1. - С. 118-126.

15. Simonov B.F., Neyman V.Y., Shabanov A.S. New conception of an electromagnetic drive for a vibration source in hole // The 18 international conference of young specialists on micro/ nanotechnologies and electron devices: Proc. - Altai, Erlagol, 29 June -3 July 2017. - P. 507-510.

16. Ивашин В.В., Певчев В.П. Определение перемещений излучателя импульсного электромагнитного источника сейсмических сигналов // Физико-технические проблемы разработки полезных ископаемых. - 2018. - № 4. - С. 51-59.

17. Haines S.S. Design and application of an electromagnetic vibrator seismic source // Journal of Environmental and Engineering Geophysics. - 2006. - V. 11 (1). - P. 9-15.

18. Sadovskii V.M., Sadovskaya O.V., Detkov V.A. Analysis of elastic waves generated in frozen grounds by means of the electromagnetic pulse source «Yenisei» // IOP Conference Series: Earth and Environmental Science. - 2018. - V. 193 (1). P. 012058.

19. Электромагнитные молоты для морского нефтегазопромыслового строительства / Б.Ф. Симонов, В.С. Вовк, Ю.В. Погарский, А.И. Кадышев // PROHЕФТЬ. Профессионально о нефти. - 2019. - № 2 (12). - С. 59-65.

20. Ряшенцев Н.П., Симонов Б.Ф., Ильинский Н.К. Электромагнитный молот для погружения свай // Газовая промышленность. - 1984. - № 11. - С. 11-15.

21. Манжесов В.К., Лукутина Н.А., Невенчанная Т.О. Динамика и синтез электромагнитных генераторов силовых импульсов. Фрунзе: Изд-во Илим, 1985. - 185 с.

22. Едыгенов Е.К. Горные машины с линейным электромагнитным двигателем // Современные проблемы теории машин. 2019. - № 8. - C. 81-84.

23. Едыгенов Е.К., Васин К.А. Результаты испытаний электромагнитного молота для безвзрывного разрушения горных пород // Горный информационно-аналитический бюллетень (научно-технический журнал). - 2020. - № 5. - С. 80-90

24. Ряшенцев Н.П., Тимошенко Е.М., Фролов А.В. Теория, расчет и конструирование электромагнитных машин ударного действия. - Новосибирск: Наука, 1970. - 259 с

25. Ижбулдин Е.А., Абрамов А.Д. Ручной электрический ударный инструмент для реализации виброударных технологий в транспортном машиностроении и строительстве // Вестник Иркутского государственного технического университета. 2017. - T. 21. - № 1 (120). - С. 32-39.
26. Strike action electromagnetic machine for immersion of rod elements into ground / K.M. Usanov, A.V. Volgin, E.A. Chetverikov, V.A. Kargin, A.P. Moiseev, Z.I. Ivanova // IOP Conference Series: Earth and Environmental Science. - 2017. V. 87. - P. 032050 .

27. Угаров Г.Г., Мошкин В.И. Перспективы развития силовых электромагнитных импульсных систем // Вестник Курганского государственного университета. - 2013. - № 2. - С. 88-90.

28. Гринченков В.П., Большенко И.А., Большенко А.В. Электромагнитный привод с низким энергопотреблением // Известия высших учебных заведений. Электромеханика. - 2015. № 5 (541). - C. $50-53$.

29. Нейман Л.А., Нейман В.Ю. Линейные синхронные электромагнитные машины для низкочастотных ударных технологий // Электротехника. - 2014. - № 12. - С. 45-49.

30. Zhao, J.H., Zhang, X.F., Zhang, J.H. Field and thrust analysis of tubular permanent magnet linear synchronous motor // Electric Machines and Control - 2010. - V. 22 (3). - P. 60-63.

31. Neyman L.A., Neyman V.Yu., Obukhov K.A. New method of the synchronous vibratory electromagnetic machine mechatronic module control // The 18 international conference of young specialists on micro/nanotechnologies and electron devices: Proc. - Altai, Erlagol, 29 June - 3 July 2017. - P. 516-519.

32. Tatevosyan A.S., Tatevosyan A.A., Zaharova N.V. Calculation of non-stationary magnetic field of the polarized electromagnet with the external attracted anchor // Journal of Physics: Conference Series. - 2018. - V. 1050 (1). - P. 012086.

33. 2D Finite Element Model for computing the electric field strengthrms generated by overhead power lines / E. Lunca, S. Vornicu, A. Salceanu, O. Bejenaru // Journal of Physics: Conference Series. - 2018. - V. 1065. - P. 1-4.

34. Shevchenko V.P., Babiychuk O.B., Boltenkov V.O. Study of current transformers magnetic field by method final elements using the FEMM software complex // Applied aspects of information technology. - 2019. - V. 2 (4). - P. 317-327.

35. Cute V.I. System of automated formation of calculating models of electric machines for FEMM software environment // Technical electrodynamics. - 2018. - № 4. - P. 74-78.

36. A high torque density outer rotor claw pole stator permanent magnet synchronous motor / J. Liang, A. Parsapour, E. Cosoroaba, M. Wu, I. Boldea, B. Fahimi // IEEE Transportation Electrification Conference and Expo (ITEC). - 2018. - P. 389-393.

37. Ряшенцев Н.П., Алабужев П.М., Никишин Н.И. Ручные электрические машины ударного действия. - М.: Недра, 1970. $198 \mathrm{c}$.

38. Черных И.В. Моделирование электротехнических устройств в MATLAB, SimPowerSystems и Simulink. - М.: ДМК Пресc; СПб.: Питер, 2008. - 288 с.

39. Kiyakli A., Hamit S. Modeling of an electric vehicle with MATLAB/Simulink // International journal of automotive science and technology. - 2018. - V. 2 (4). - P. 9-15.

40. Буль О.Б. Методы расчета магнитных систем электрических аппаратов: Магнитные цепи, поля и программа FEMM. - М.: ИЦ «Академия», 2005. - $336 \mathrm{c.}$

41. Нейман Л.А., Нейман В.Ю. Моделирование динамических процессов в электромагнитных преобразователях энергии для систем генерирования силовых воздействий и низкочастотных вибраций // Известия Томского политехнического университета. Инжиниринг георесурсов. - 2015. - Т. 326. - № 4. C. $154-162$.

Поступила 31.08.2020 2.

\section{Информация об авторах}

Нейман Л.А., доктор технических наук, профессор кафедры электротехнических комплексов Новосибирского государственного технического университета.

Нейман В.Ю., доктор технических наук, профессор, заведующий кафедрой теоретических основ электротехники Новосибирского государственного технического университета. 


\title{
GENERALIZED MODEL OF A TWO-COIL SYNCHRONOUS ELECTROMAGNETIC MACHINE FOR VIBRATORY IMPACT TECHNOLOGICAL SYSTEMS
}

\author{
Lyudmila A. Neyman', \\ neyman31@gmail.com \\ Vladimir Yu. Neyman ${ }^{1}$, \\ nv.nstu@ngs.ru \\ 1 Novosibirsk State Technical University, \\ 20, Karl Marks avenue, Novosibirsk, 630073, Russia.
}

\begin{abstract}
Relevance of the research is explained by the perspective of electric pulse systems based on impact and vibratory impact linear electromagnetic machines application in geological exploration, mining operations and minerals processing technologies. Impact synchronous electromagnetic machines are useful for energy saving. Impact force pulse frequency of these machines is equal to or multiple of the power source frequency. The main advantage of devices and equipment based on impact and vibratory impact synchronous electromagnetic machines is the capability of power consumption decrease in near-resonant modes. These modes study is important for the design of exact dynamic models of different variants of impact synchronous electromagnetic machines operating only in transient modes.

The aim of the research is to develop the generalized mathematical model of a two-coil impact synchronous machine with an electromagnetic drive for vibratory impact technological systems. The model simplifies the mathematical description of known variants machine schemes when carrying out dynamic calculations.

The methods used in the research include differential equations of the electrical equilibrium and mechanical interaction of directly moving masses, Lagrange's equation of the second kind, finite-element methods of magnetic field modeling, methods of structured modeling in Matlab Simulink, design and experimental characteristics comparison.

Results. The authors have proposed the generalized design model of the multi-mass two-coil impact synchronous electromagnetic machine for its known and new variants. The differential equations system were derived for this model. The generalized model is the basis of dynamic models of different variants of impact synchronous electromagnetic machines. The paper considers as an example the dynamic model of the four-mass two-coil impact synchronous electromagnetic machine with the striker elastic reverse. The simulation results stated for the operating mode include the oscillograms of the current in the coils, the striker motion and velocity illustrating switching on and reaching the quasi-stationary operating mode. It follows from integral characteristics comparison that the difference between the design and experimental data is $4 \ldots .6 \%$ and it is no more than common engineering design error. The proposed generalized model helps effectively to simplify the mathematical description of the impact synchronous electromagnetic motor and decrease the time interval for model implementation.
\end{abstract}

Key words: Electrical impulse system, linear synchronous machine, synchronous frequency, number of degrees of freedom, generalized model, dynamic model, simulation.

\section{REFERENCES}

1. Ashavskiy A.M., Volpert A.Ya., Shneynbaum V.S. Silovye impulsnye sistemy [Power impulse systems]. Moscow, Mashinostroenie Publ., 1978. 200 p.

2. Abramov A.D., Tyunyukova T.K., Izhbuldin E.A. Vibroudarnye tekhnologii dlya stroitelno-dorozhnykh mashin [Vibration shock technologies for road-building machines]. Mir transporta, 2016, vol. 14, no. 4 (65), pp. 62-69.

3. Abramov A.D., Galay M.S. Perspektivy primeneniya mashin udarnogo deystviya pri uprochnenii svarnykh shvov [Prospects for the use of impact machines for strengthening welded seams]. Fundamentalnye i prikladnye voprosy gornykh nauk, 2016, vol. 2, no. 3, pp. 7-10.

4. Moshkin V.I. Pulsing linear electromagnetic motor mechanical work within the desaturated magnetic system. International Conference on Industrial Engineering, Applications and Manufacturing, ICIEAM. 2017. pp. 8076319.

5. Neiman V.Y. Integrated linear electromagnetic motors for pulsed technologies. Russian Electrical Engineering, 2003, vol. 74, no. 9, pp. 30-35. In Rus.

6. Wu T., Tang Y., Tang S., Li Y., He W., Chen E. Design and analysis of a new down-the-hole electromagnetic hammer driven by tube linear motor. IET Electric Power Applications, 2017, vol. 11, no. 9, pp. 1558-1565.

7. Usanov K.M., Volgin A.V., Kargin V.A., Moiseev A.P., Chetverikov E.A. Electric converters of electromagnetic strike machine with battery power. IOP Conference Series: Materials Science and Engineering, 2018, vol. 327, pp. 052032.
8. Kargin V.A., Tikhomirova L.B., Abramov A.D., Galai M.S. Effect of vibroimpact treatment on the physical and mechanical properties of the surface layer of welded joints in rails. Welding International, 2014, vol. 28, no. 3, pp. 245-247.

9. Neyman L.A., Neyman V.Yu., Shabanov A.S. Vibration dynamics of an electromagnetic drive with a half-period rectifier. The 18 international conference of young specialists on micro/nanotechnologies and electron devices: proc. Altai, Erlagol, 29 June -3 July 2017. pp. 503-506.

10. Edygenov E.K., Lyashkov V. Electromagnetic rock breaker for non-explosive rocks breaking. International Symposium on planning of mining and selection of equipment of MPES 2011. Almata, 2011. pp. 1002-1012.

11. Pavlov V.E. Issledovanie rezhimov raboty elektromagnitnogo molota [Research of operating modes of the electromagnetic hammer]. Vestnik Irkutskogo gosudarstvennogo tekhnicheskogo universiteta, 2017, vol. 21, no. 12 (131), pp. 164-173.

12. Ugarov G.G., Neiman V.Y. Evaluation of operating conditions for electromagnetic impactors. Journal of Mining Science, 1996, vol. 32, no. 4, pp. 305-312. In Rus.

13. Simonov B.F., Oparin V.N., Kordubaylo A.O., Vostrikov V.I. Eksperimentalnye issledovaniya effektivnosti izlucheniya ot skvazhinnogo impulsnogo vibroistochnika [Experimental studies of the efficiency of radiation from a borehole pulsed vibration source]. Gorny informatsionno-analiticheskiy byulleten (nauchnotekhnicheskiy zhurnal), 2019, no. 8, pp. 180-189.

14. Simonov B.F., Neiman V.Y., Shabanov A.S. Pulsed linear solenoid actuator for deep-well vibration source. Journal of Mining Science, 2017, vol. 51, no. 1, pp. 117-125. In Rus. 
15. Simonov B.F., Neyman V.Y., Shabanov A.S. New conception of an electromagnetic drive for a vibration source in hole. The 18 international conference of young specialists on micro/nanotechnologies and electron devices: proc. Altai, Erlagol, 29 June -3 July 2017. pp. 507-510.

16. Ivashin V.V., Pevchev V.P. Determining displacements of pulsed electromagnetic source of seismic signals. Journal of Mining Science, 2018, vol. 54, no. 4, pp. 575-581. In Rus.

17. Haines S.S. Design and application of an electromagnetic vibrator seismic source. Journal of Environmental and Engineering Geophysics, 2006, vol. 11 (1), pp. 9-15.

18. Sadovskii V.M., Sadovskaya O.V., Detkov V.A. Analysis of elastic waves generated in frozen grounds by means of the electromagnetic pulse source «Yenisei». IOP Conference Series. Earth and Environmental Science, 2018, vol. 193 (1), pp. 012058.

19. Simonov B.F., Vovk V.S., Pogarskiy Yu.V., Kadyshev A.I. Elektromagnitnye moloty dlya morskogo neftegazopromyslovogo stroitelstva [Electromagnetic hammers for offshore oil and gas field construction]. PRONEFT. Professionalno o nefti, 2019, no. 2 (12), pp. 59-65.

20. Ryashentsev N.P., Simonov BF., Ilinskiy N.K. Elektromagnitny molot dlya pogruzheniya svay [Electromagnetic hammer for driving piles]. Gazovaya promyshlennost, 1984, no. 11, pp. 11-15.

21. Manzhesov V.K., Lukutina N.A., Nevenchannaya T.O. Dinamika sintez elektromagnitnykh generatorov silovykh impulsov [Dynamics and synthesis of electromagnetic power pulse generators]. Frunze, Ilim Publ., 1985. 185 p.

22. Edygenov E.K. Gornye mashiny s lineynym elektromagnitnym dvigatelem [Mining machines with linear electromagnetic motor] Sovremennye problemy teorii mashin, 2019, no. 8, pp. 81-84.

23. Edygenov E.K., Vasin K.A. Rezultaty ispytaniy elektromagnitnogo molota dlya bezvzryvnogo razrusheniya gornykh porod [Test results of an electromagnetic hammer for non-explosive destruction of rocks]. Gorny informatsionnoanaliticheskiy byulleten (nauchno-tekhnicheskiy zhurnal), 2020, no. 5 , pp. $80-90$.

24. Ryashentsev N.P., Timoshenko E.M., Frolov A.V. Teoriya raschet $i$ konstruirovanie elektromagnitnykh mashin udarnogo deystviya [Theory, calculation and design of electromagnetic impact machines]. Novosibirsk, Nauka Publ., 1970. 259 p.

25. Izhbuldin E.A., Abramov A.D. Ruchnoy elektricheskiy udarny instrument dlya realizatsii vibroudarnykh tekhnologiy v transportnom mashinostroenii i stroitelstve [Hand-held electric percussion tool for the implementation of vibration shock technologies in transport engineering and construction]. Vestnik Irkutskogo gosudarstvennogo tekhnicheskogo universiteta, 2017, vol. 21, no. 1 (120), pp. 32-39

26. Usanov K.M., Volgin A.V., Chetverikov E.A., Kargin V.A., Moiseev A.P., Ivanova Z.I. Strike action electromagnetic machine for immersion of rod elements into ground. IOP Conference Series: Earth and Environmental Science, 2017, vol. 87, pp. 032050.

27. Ugarov G.G., Moshkin V.I. Perspektivy razvitiya silovykh elektromagnitnykh impulsnykh sistem [Prospects for the development of power electromagnetic pulse systems]. Vestnik Kurganskogo gosudarstvennogo universiteta, 2013, no. 2, pp. 88-90.

28. Grinchenkov V.P., Bolshenko I.A., Bolshenko A.V Elektromagnitny privod s nizkim energopotrebleniem [Low power consumption electromagnetic actuator]. Izvestiva vysshikh uchebnykh zavedeniy. Elektromekhanika, 2015, no. 5 (541), pp. 50-53.

29. Neiman L.A. Neiman V.Yu. Linear synchronous electromagnetic machines for lowfrequency impact technologies. Russian Electrical Engineering, 2014, vol. 85, no. 12, pp. 752-756. In Rus.

30. Zhao J.H., Zhang X.F., Zhang J.H. Field and thrust analysis of tubular permanent magnet linear synchronous motor. Electric Machines and Control, 2010, vol. 22 (3), pp. 60-63.

31. Neyman L.A., Neyman V.Yu., Obukhov K.A. New method of the synchronous vibratory electromagnetic machine mechatronic module control. The 18 international conference of young specialists on micro/nanotechnologies and electron device: proc. Altai, Erlagol, 29 June - 3 July 2017. pp. 516-519.

32. Tatevosyan A.S., Tatevosyan A.A., Zaharova N.V. Calculation of non-stationary magnetic field of the polarized electromagnet with the external attracted anchor. Journal of Physics: Conference Series, 2018, vol. 1050 (1), pp. 012086.

33. Lunca E., Vornicu S., Salceanu A., Bejenaru O. 2D Finite Element Model for computing the electric field strength-rms generated by overhead power lines. Journal of Physics: Conference Series, 2018, vol. 1065, pp. 1-4.

34. Shevchenko V.P., Babiychuk O.B., Boltenkov V.O. Study of current transformers magnetic field by method final elements using the FEMM software complex. Applied aspects of information technology, 2019, vol. 2 (4), pp. 317-327.

35. Cute V.I. System of automated formation of calculating models of electric machines for FEMM software environment. Technical electrodynamics, 2018, no. 4, pp. 74-78.

36. Liang J., Parsapour A., Cosoroaba E., Wu M., Boldea I., Fahimi B. A high torque density outer rotor claw pole stator permanent magnet synchronous motor. IEEE Transportation Electrification Conference and Expo (ITEC), 2018. pp. 389-393.

37. Ryashentsev N.P., Alabuzhev P.M., Nikishin N.I. Ruchnye elektricheskie mashiny udarnogo deystviya [Manual electric impact machines]. Moscow, Nedra Publ., 1970. 198 p.

38. Chernykh I.V. Modelirovanie elektrotekhnicheskikh ustroystv v MATLAB, SimPowerSystems i Simulink [Simulation of electrical devices in MATLAB, SimPowerSystems and Simulink]. Moscow, DMK Press; St. Petersburg, Piter Publ., 2008. 288 p.

39. Kiyakli A., Hamit S. Modeling of an electric vehicle with MATLAB/Simulink. International journal of automotive science and technology, 2018, vol. 2, no. 2, pp. 9-15.

40. Bul O.B. Metody rascheta magnitnykh sistem elektricheskikh apparatov: Magnitnye tsepi, polya i programma FEMM [Methods for calculating the magnetic systems of electrical devices: Magnetic circuits, fields and the FEMM program]. Moscow, Akademiya Publ., 2005. 336 p.

41. Neyman L.A., Neyman V.Yu. Simulation of dynamic processes in electromagnetic energy converters for force effects and lowvfrequency vibrations generation systems. Bulletin of the Tomsk Polytechnic University. Geo Assets Engineering, 2015, vol. 326, no. 4, pp. 154-162. In Rus.

Received: 31 August 2020.

\section{Information about the authors}

Lyudmila A. Neyman, Dr. Sc., professor, Novosibirsk State Technical University.

Vladimir Yu. Neyman, Dr. Sc., professor, Novosibirsk State Technical University. 